\title{
Rational Points of Some Elliptic Curves Related to the Tilings of the Equilateral Triangle
}

\section{Miklós Laczkovich ${ }^{1}$}

Received: 3 February 2019 / Revised: 7 October 2019 / Accepted: 9 October 2019 /

Published online: 22 October 2019

(c) The Author(s) 2019

\begin{abstract}
Let $n$ be a positive and squarefree integer. We show that the equilateral triangle can be dissected into $n \cdot k^{2}$ congruent triangles for some $k$ if and only if $n \leq 3$, or at least one of the curves $C_{n}: y^{2}=x(x-n)(x+3 n)$ and $C_{-n}: y^{2}=x(x+n)(x-3 n)$ has a rational point with $y \neq 0$. We prove that if $p$ is a positive prime such that $p \equiv 7$ $(\bmod 24)$, then $C_{p}$ and $C_{-p}$ do not have such points. Consequently, for these primes the equilateral triangle cannot be dissected into $p \cdot k^{2}$ congruent triangles for any $k$.
\end{abstract}

Keywords Tilings of the equilateral triangle $\cdot$ Rank of some elliptic curves over the rationals

\section{Introduction and Main Results}

Let $C_{n}$ denote the elliptic curve $y^{2}=x(x-n)(x+3 n)$, where $n$ is an integer. The group of rational points of $C_{n}$ will be denoted by $\Gamma_{n}$. We say that $(x, y) \in C_{n}$ is a nontrivial rational point of $C_{n}$ if $x, y$ are nonzero rational numbers; that is, if the order of $(x, y)$ as an element of the group $\Gamma_{n}$ is greater than two. Our first result shows that the existence of nontrivial rational points of $C_{n}$ is closely related to the number of pieces in certain tilings of the equilateral triangle.

Theorem 1.1 For every positive and squarefree integer $n$ the following are equivalent.

(i) There is a positive integer $k$ such that the equilateral triangle can be dissected into $n \cdot k^{2}$ congruent triangles.

(ii) Either $n \leq 3$, or at least one of the curves $C_{n}$ and $C_{-n}$ has a nontrivial rational point.

\section{Dedicated to the memory of Ricky Pollack.}

Miklós Laczkovich

miklos.laczkovich@gmail.com

1 Eötvös Loránd University, Budapest, Hungary 
The proof of Theorem 1.1 is based on the fact that the congruent copies of a triangle with sides $a, b, c$ and corresponding angles $\alpha, \beta, \gamma$ tile an equilateral triangle if and only if either $\alpha, \beta, \gamma$ are multiples of $\pi / 6$, or $\gamma \in\{\pi / 3,2 \pi / 3\}$ and $a, b, c$ are pairwise commensurable (see [4, Thm. 3.3]). By the law of cosines, we have $\gamma=\pi / 3$ or $2 \pi / 3$ if and only if $c^{2}=a^{2}+b^{2} \pm a b$. Such triples are, e.g., $(a, b, c)=(7,8,13)$ or $(a, b, c)=(3,5,7)$.

Suppose that $a, b, c$ are positive integers with $c^{2}=a^{2}+b^{2} \pm a b$. Then the triangle with sides $a, b, c$ tiles an equilateral triangle $T$. If the side length of $T$ is $m$ and the tiling has $N$ pieces, then, comparing the areas we get $m^{2}=N \cdot a b$, and thus the square free part of $N$ is the same as that of $a b$. For example, if $(a, b, c)=(7,8,13)$, then the construction described in [3, Thm. 3.1] produces a tiling with $2,469,600=14 \cdot 420^{2}$ pieces. For the triangle with sides $3,5,7$, a tiling with $10,935=15 \cdot 27^{2}$ pieces was found by Michael Beeson (see [2, Fig. 22, p. 28]).

As we shall see, a simple transformation maps these triples into nontrivial rational points of one of the corresponding curves $C_{n}$ or $C_{-n}$. Thus the triple $(7,8,13)$ gives the point $(-6,48)$ of $C_{-14}$, and $(3,5,7)$ gives the point $(-5,50)$ of $C_{-15}$.

In the other direction, every nontrivial rational point of $C_{n}$ or $C_{-n}$ determines a triple $(a, b, c)$ as above. For example, from the point $(-1,8)$ of $C_{-5}$ we obtain the triple $(5,16,19)$, and the from the point $(-1,30)$ of $C_{17}$ we get $(17,225,217)$. The proof of Theorem 1.1 will be given in the next section.

Remarks 1.2 1. Since every triangle $\Delta$ can be dissected into $m^{2}$ congruent triangles similar to $\Delta$ for every $m$, it is clear that (i) of Theorem 1.1 is equivalent to the following statement.

(i') There are infinitely many positive integers $k$ such that the equilateral triangle can be dissected into $n \cdot k^{2}$ congruent triangles.

2. We shall prove in Lemma 3.1 that if $p$ is a positive prime, then the only torsion points of $\Gamma_{p}$ and $\Gamma_{-p}$ are the points having zero $y$-coordinates. Therefore, if $n$ is a positive prime, then (ii) of Theorem 1.1 is equivalent to the following statement.

(ii') Either $n \leq 3$, or at least one of the groups $\Gamma_{n}$ and $\Gamma_{-n}$ has positive rank.

It is easy to see that if $n, k$ are nonzero integers then $C_{n}$ has a nontrivial rational point if and only if $C_{n k^{2}}$ has one. Therefore, we have the following corollary of Theorem 1.1 .

Corollary 1.3 If the equilateral triangle can be dissected into $N$ congruent triangles, then either $N=k^{2}, N=2 k^{2}$ or $N=3 k^{2}$ for some $k$, or at least one of the curves $C_{N}$ and $C_{-N}$ has a nontrivial rational point.

We remark that the converse is not true. For example, $(-1,8)$ is a nontrivial rational point of $C_{-5}$, but the equilateral triangle cannot be dissected into 5 congruent triangles. This follows from a result of Beeson stating that the equilateral triangle cannot be dissected into $p$ congruent triangles for any prime $p>3$ (see [1]). On the other hand, the equilateral triangle can be dissected into $5 k^{2}$ congruent triangles for infinitely many positive integer $k$ by Theorem 1.1 .

In Sect. 3 we shall prove that if $p$ is a positive prime and $p \equiv 7(\bmod 24)$, then the curves $C_{p}$ and $C_{-p}$ have no nontrivial rational points (see Corollary 3.6). Comparing with Theorem 1.1 we obtain the following. 
Corollary 1.4 If $p$ is a positive prime such that $p \equiv 7(\bmod 24)$, then the equilateral triangle cannot be dissected into $p \cdot k^{2}$ congruent triangles for any $k$.

\section{Proof of Theorem 1.1}

(i) $\Rightarrow$ (ii): Suppose that the equilateral triangle $T$ can be tiled with $n \cdot k^{2}$ congruent triangles having angles $\alpha, \beta, \gamma$ and corresponding sides $a, b, c$. We may assume that the sides of $T$ equal 1 .

By [4, Thm. 3.3], one of the following cases holds: $\alpha=\beta=\pi / 6$ and $\gamma=$ $2 \pi / 3 ; \alpha=\pi / 6, \beta=\pi / 2, \gamma=\pi / 3 ; \gamma \in\{\pi / 3,2 \pi / 3\}$ and $a, b, c$ are pairwise commensurable.

Comparing the areas of $T$ and the tiles we obtain $n k^{2} \cdot a b \cdot \frac{\sqrt{3}}{4}=\frac{\sqrt{3}}{4}$; that is,

$$
n k^{2} \cdot a b=1
$$

If $\alpha=\beta=\pi / 6$, then $a=b$ and thus, by (1), $a=b=1 /(k \cdot \sqrt{n})$. By $c / a=\sqrt{3}$ we have $c=\sqrt{3} /(k \cdot \sqrt{n})$. Since the side of the equilateral triangle is tiled with segments of length $a$ and $c$, we obtain $1=r a+s c$ with suitable nonnegative integers $r, s$. Thus $r+s \sqrt{3}=k \cdot \sqrt{n}$. Since $n$ is squarefree, this implies $n=1$ or $n=3$.

If $\alpha=\pi / 6, \beta=\pi / 2$ and $\gamma=\pi / 3$, then $b=2 a$ and thus, by (1), $a=1 /(k \cdot \sqrt{2 n})$. By $c / a=\sqrt{3}$ we have $c=\sqrt{3} /(k \cdot \sqrt{2 n})$. The side of the equilateral triangle is tiled with segments of length $a, 2 a$ and $c$, hence $1=r a+s c$ with suitable nonnegative integers $r, s$. Thus $r+s \sqrt{3}=k \cdot \sqrt{2 n}$. Since $n$ is squarefree, this implies $n=2$ or $n=6$. Now $(9,27)$ is a point of $C_{6}: y^{2}=x(x-6)(x+18)$, and thus the statement of (ii) is true in these cases.

In the remaining cases $a, b, c$ are pairwise commensurable, and $\gamma=\pi / 3$ or $\gamma=$ $2 \pi / 3$. Then we have $c^{2}=a^{2}+b^{2} \pm a b$ by the law of cosines. Since $q a+r b+s c=1$ with nonnegative integers $q, r, s$, it follows that $a, b, c$ are rational. Replacing $a$ by $-a$ if necessary, we may assume $c^{2}=a^{2}+b^{2}+a b$. Under this change (1) becomes $\pm n k^{2} \cdot a b=1$. We put $t=(c-b) / a$; then $t$ is rational, and $b=c-t a$. We have

$$
\begin{aligned}
c^{2} & =a^{2}+b^{2}+a b=a^{2}+(c-t a)^{2}+a c-t a^{2} \\
& =a^{2}\left(t^{2}-t+1\right)-2 a c t+a c+c^{2},
\end{aligned}
$$

$a^{2}\left(t^{2}-t+1\right)=a c(2 t-1)$, and $a / c=(2 t-1) / d$, where $d=t^{2}-t+1$. Note that $d \neq 0$, as the polynomial $X^{2}-X+1$ has no rational roots. Then we have $b / c=1-(t a / c)=\left(1-t^{2}\right) / d$. From (1) we get

$$
1= \pm n k^{2} a b= \pm n \cdot(2 t-1)\left(1-t^{2}\right) \cdot(c k / d)^{2}
$$

and $(2 t-1)\left(t^{2}-1\right)=\mp n v^{2}$, where $v=d /(n k c)$ is a nonzero rational number.

Putting $x=n(2 t-1)$ we get $t=(x+n) /(2 n), t-1=(x-n) /(2 n), t+1=$ $(x+3 n) /(2 n)$, and

$$
x(x-n)(x+3 n)=(2 t-1)\left(t^{2}-1\right) \cdot 4 n^{3}=\mp n v^{2} \cdot 4 n^{3}=\mp y^{2},
$$


where $y=2 n^{2} v$. Therefore, either $(x, y)$ is a point of $C_{n}$ or $(-x, y)$ is a point of $C_{-n}$. (ii) $\Rightarrow$ (i): It is clear that if $n \leq 3$ then the equilateral triangle can be dissected into $n$ congruent triangles.

Suppose that $x, y$ are rational numbers, $y \neq 0$, and $(x, y)$ is a rational point of either $C_{n}$ or $C_{-n}$. Then one of $t=x / n$ and $t=-x / n$ satisfies $t(t+1)(t-3)= \pm y^{2} / n^{3}$. Fix such a $t$. Note that $t \neq 0,-1,3$. Putting $a=4 t, b=t^{2}-2 t-3$ and $c=t^{2}+3$ we have $a b \neq 0$ and $a^{2}+b^{2}+a b=c^{2}$. Then $|a|,|b|, c$ are the sides of a rational triangle $\Delta$ such that $a^{2}+b^{2} \pm|a| \cdot|b|=c^{2}$, and thus, by the law of cosines, the angle between the sides of length $|a|$ and $|b|$ equals $\pi / 3$ or $2 \pi / 3$. By [3, Thm. 3.1], there is an equilateral triangle $T$ that can be dissected into triangles congruent to $\Delta$. Let $m$ be the length of the side of $T$, and let $N$ be the number of pieces of the decomposition. Then $N|a b|=m^{2}$, hence

$$
m^{2} / N=|a b|=4\left|t\left(t^{2}-2 t-3\right)\right|=4|t(t+1)(t-3)|=4 y^{2} / n^{3}
$$

and $N=n^{3} m^{2} /\left(4 y^{2}\right)=n k^{2}$, where $k=n m /(2 y)$. Now $k$ is rational and $n$ is squarefree by assumption, so $N=n k^{2}$ implies that $k$ must be an integer. We have found a dissection of $T$ into $n \cdot k^{2}$ congruent triangles, proving (i).

\section{Rational Points of $C_{ \pm p}$}

In this section we show that if $p$ is a positive prime and $p \equiv 7(\bmod 24)$, then $C_{p}$ and $C_{-p}$ have no nontrivial rational points (see Corollary 3.6). Recall that the group of rational points of $C_{n}$ is denoted by $\Gamma_{n}$.

Lemma 3.1 Let $p$ be a positive prime. Then the torsion points of the group $\Gamma_{p}$ are the points $(0,0),(p, 0),(-3 p, 0)$ and $\mathcal{O}$ (the point at infinity). The torsion points of $\Gamma_{-p}$ are the points $(0,0),(-p, 0),(3 p, 0)$ and $\mathcal{O}$.

Proof The points listed above, being of order two and one, are torsion points. Suppose there exists another torsion point $(x, y)$. Since the discriminant of the curves equals $p^{2} \cdot(3 p)^{2} \cdot(4 p)^{2}=3^{2} \cdot 2^{4} \cdot p^{6}$, it follows from the Nagell-Lutz theorem that $x, y \in \mathbb{Z}$, $y \neq 0$ and $y \mid 3 \cdot 2^{2} \cdot p^{3}$. We distinguish between two cases.

Case I: $p \mid y$. Then $p\left|x, x=p z, p^{2}\right| y, y=p^{2} u, u \neq 0$, and

$$
p u^{2}=z(z \mp 1)(z \pm 3) \text {. }
$$

Clearly, $z \geq-2$. It is easy to check that if $-2 \leq z \leq 13$ then $z(z \mp 1)(z \pm 3)$ is not of the form $q u^{2}$, where $q$ is prime and $u \neq 0$, except when $z=4$ and $z(z+1)(z-3)=5 \cdot 2^{2}$. This gives the point $P_{1}=(20,50)$ of $\Gamma_{-5}$. One can easily check that the $x$-coordinate of $2 P_{1}$ is not an integer, hence $P_{1}$ is not a torsion point. (Thus $\Gamma_{-5}$ has positive rank.) Therefore, we may assume $z \geq 14$.

If $p=2$ or $p=3$ then $y=p^{2} u \mid 3 \cdot 2^{2} \cdot p^{3}$ implies that all prime factors of $z$ and $z \pm 1$ are 2 and 3 . Thus $z=2^{\alpha}, z \pm 1=3^{\beta}$ or the other way around. Then $z \leq 10$ which is impossible. 
Therefore, we may assume $p>3$. Then at most one of the terms $z, z \mp 1, z \pm 3$ is divisible by $p$. Since $u \mid 3 \cdot 2^{2} \cdot p^{3}$, it follows from (2) that the product of two of the terms $z, z \mp 1 z \pm 3$ is a divisor of $3^{2} \cdot 2^{4}=144$. By $z \geq 4$ this implies $z(z-3) \leq 144$, hence $z \leq 13$ which is impossible.

Case II: $p \nmid y$. Then $y \mid 12$. Replacing $x$ by $-x$ if necessary, we have $x(x+p)(x-$ $3 p)= \pm y^{2}$, and thus

$$
|x(x+p)(x-3 p)|=y^{2} \mid 144 .
$$

It is easy to see that if $a$ is a positive integer and $x$ is an integer different from 0 and $a$, then $|x(a-x)| \geq a-1$. Therefore, $|x(x+p)| \geq p-1,|x(x-3 p)| \geq 3 p-1$, $|(x+p)(x-3 p)| \geq 4 p-1$,

$$
(p-1)(3 p-1)(4 p-1) \leq|x(x+p)(x-3 p)|^{2} \leq 144^{2},
$$

and thus $p \leq 11$.

It follows from (3) that there are (positive or negative) divisors $d_{1}, d_{2}$ of 144 such that $d_{2}-d_{1}=4 p,\left|x \cdot d_{1} \cdot d_{2}\right|$ is a square and is a divisor of 144 , where $x=d_{2}-p$. Checking the cases $p=2,3,5,7,11$, we find that the only possibility is $p=5$, $\left(d_{1}, d_{2}\right)=(-16,4)$ and $x=-1$. This gives the point $P_{2}=(-1,8)$ of $\Gamma_{-5}$. One can easily check that $P_{2}=P_{1}+P_{0}$, where $P_{0}=(-5,0)$ and $P_{1}=(20,50)$. Since $P_{0}$ is a torsion point of $\Gamma_{-5}$ and $P_{1}$ is not, it follows that $P_{2}$ is not a torsion point either.

\section{Theorem 3.2}

(i) The rank of $\Gamma_{p}$ is at most two for every positive prime $p$.

(ii) If $p \not \equiv 1(\bmod 24)$, then the rank of $\Gamma_{p}$ is at most one.

(iii) If $p=2, p=3$ or $p \equiv 5,7$ or $19(\bmod 24)$, then the rank of $\Gamma_{p}$ is zero.

In the proof of Theorem 3.2 we apply the method described in $[5, \S 5$, Chap. III, pp.92-94]. Consider the curves

$$
C_{p}: y^{2}=x^{3}+2 p x^{2}-3 p^{2} x \text { and } \bar{C}_{p}: y^{2}=x^{3}-4 p x^{2}+16 p^{2} x
$$

with groups of rational points $\Gamma_{p}=C_{p}(\mathbb{Q})$ and $\bar{\Gamma}_{p}=\bar{C}_{p}(\mathbb{Q})$. We define $\alpha: \Gamma_{p} \rightarrow$ $\mathbb{Q}^{*} / \mathbb{Q}^{* 2}$ by $\alpha(\mathcal{O})=1, \alpha(0,0)=-3 p^{2} \equiv-3$ and, for $x \neq 0, \alpha(x, y)=x(\bmod$ $\left.\mathbb{Q}^{* 2}\right)$. Then $\alpha$ is a homomorphism from $\Gamma_{p}$ into $\mathbb{Q}^{*} / \mathbb{Q}^{* 2}$.

We also define $\bar{\alpha}: \bar{\Gamma}_{p} \rightarrow \mathbb{Q}^{*} / \mathbb{Q}^{* 2}$ by $\bar{\alpha}(\mathcal{O})=1, \bar{\alpha}(0,0)=16 p^{2} \equiv 1$ and, for $x \neq 0, \alpha(x, y)=x\left(\bmod \mathbb{Q}^{* 2}\right)$. Then $\bar{\alpha}$ is a homomorphism from $\bar{\Gamma}_{p}$ into $\mathbb{Q}^{*} / \mathbb{Q}^{* 2}$. The rank $r$ of $\Gamma_{p}$ satisfies

$$
2^{r}=\frac{\# \alpha\left(\Gamma_{p}\right) \cdot \# \bar{\alpha}\left(\bar{\Gamma}_{p}\right)}{4}
$$


(see [5, p.91]). Here $\alpha\left(\Gamma_{p}\right)$ equals the set of divisors $b_{1}$ of $b=-3 p^{2}\left(\bmod \mathbb{Q}^{* 2}\right)$ such that the equation

$$
N^{2}=b_{1} M^{4}+2 p M^{2} e^{2}+\left(-3 p^{2} / b_{1}\right) e^{4}
$$

is solvable in pairwise coprime integers $N, M, e$ satisfying $M \neq 0$ and $\operatorname{gcd}\left(e, b_{1}\right)=$ $\operatorname{gcd}\left(M,-3 p^{2} / b_{1}\right)=1$ (see [5, pp. 92-93]). Similarly, $\alpha\left(\bar{\Gamma}_{p}\right)$ equals the set of divisors $b_{1}$ of $\bar{b}=16 p^{2}\left(\bmod \mathbb{Q}^{* 2}\right)$ such that the equation

$$
N^{2}=b_{1} M^{4}-4 p M^{2} e^{2}+\left(16 p^{2} / b_{1}\right) e^{4}
$$

is solvable in pairwise coprime integers $N, M, e$ satisfying $M \neq 0$ and $\operatorname{gcd}\left(e, b_{1}\right)=$ $\operatorname{gcd}\left(M, 16 p^{2} / b_{1}\right)=1$.

The statement of Theorem 3.2 is an immediate consequence of (4) and of the following lemma.

\section{Lemma 3.3}

(i) $\# \alpha\left(\Gamma_{p}\right) \leq 8$ for every positive prime $p$.

(ii) If $p=2, p=3$ or $p \equiv 5,7,13$ or $19(\bmod 24)$, then $\# \alpha\left(\Gamma_{p}\right) \leq 4$.

(iii) $\# \alpha\left(\bar{\Gamma}_{p}\right) \leq 2$ for every positive prime $p$.

(iv) If $p \not \equiv 1(\bmod 12)$, then $\# \alpha\left(\bar{\Gamma}_{p}\right)=1$.

\section{Proof}

(i) is obvious from $b_{1} \in\{ \pm 1, \pm 3, \pm p, \pm 3 p\}\left(\bmod \mathbb{Q}^{* 2}\right)$.

(ii) If $p=3$ then $b_{1} \in\{ \pm 1, \pm 3\}\left(\bmod \mathbb{Q}^{* 2}\right)$, and $\# \alpha\left(\Gamma_{p}\right) \leq 4$. Therefore, we may assume $p \neq 3$. We have $(p, 0),(-3 p, 0) \in \Gamma_{p}$ and $\alpha(0,0)=-3 p^{2} \equiv-3$, and thus $1, p,-3,-3 p \in \alpha\left(\Gamma_{p}\right)$. Since $\alpha\left(\Gamma_{p}\right)$ is a subgroup of $\mathbb{Q}^{*} / \mathbb{Q}^{* 2}$, it follows that $\# \alpha\left(\Gamma_{p}\right)$ equals 4 or 8 , and it equals 8 if and only if $-1 \in \alpha\left(\Gamma_{p}\right)$.

Suppose that $\# \alpha\left(\Gamma_{p}\right)=8$. Then $-1 \in \alpha\left(\Gamma_{p}\right)$ and thus, by $b_{1} \mid 3 p^{2},(5)$ is solvable for at least one of $b_{1}=-1$ and $b_{1}=-p^{2}$.

Suppose that $N^{2}=-M^{4}+2 p M^{2} e^{2}+3 p^{2} e^{4}$ is solvable. If $p=2$, then $M$ is odd by $\operatorname{gcd}\left(M, 3 p^{2}\right)=1$, and $N^{2} \equiv-M^{4}(\bmod 4)$, which is impossible. If $p>3$, then $p \nmid M$ by $\operatorname{gcd}\left(M, 3 p^{2}\right)=1$, and thus we have $\left(\frac{-1}{p}\right)=1$ and $p \equiv 1(\bmod 4)$.

We have $N^{2}=\left(3 p e^{2}-M^{2}\right)\left(p e^{2}+M^{2}\right)=A \cdot B$. Since $p \nmid M$ and $\operatorname{gcd}(M, e)=1$, it follows that $\operatorname{gcd}(A, B) \mid 4$. If $\operatorname{gcd}(A, B)=1$ or 4 , then $A$ and $B$ are squares. Thus $3 p e^{2}-M^{2}=n^{2}$, hence $-M^{2} \equiv n^{2}(\bmod 3)$, which is impossible, as $3 \nmid M$.

If $\operatorname{gcd}(A, B)=2$, then $A / 2$ and $B / 2$ are squares. Thus $3 p e^{2}-M^{2}=2 n^{2}$, hence $-M^{2} \equiv 2 n^{2}(\bmod p)$. Since $p \nmid M$ and $p \equiv 1(\bmod 4)$, we get $\left(\frac{2}{p}\right)=1$ and $p \equiv 1$ $(\bmod 8)$.

Next suppose that $N^{2}=-p^{2} M^{4}+2 p M^{2} e^{2}+3 e^{4}$ is solvable. Then we have $\operatorname{gcd}(M, 3)=1$. If $p=2$, then $e$ is odd (since otherwise both $N$ and $e$ would be even), and $N^{2} \equiv 3 e^{4}(\bmod 4)$, which is impossible. Suppose $p>3$. Then $p \nmid e$ (since otherwise both $e$ and $N$ would be divisible by $p)$, and thus $\left(\frac{3}{p}\right)=1$ and $p \equiv \pm 1(\bmod$ 12). 
We have $N^{2}=\left(3 e^{2}-p M^{2}\right)\left(e^{2}+p M^{2}\right)=C \cdot D$. Since $p \nmid e$ and $\operatorname{gcd}(M, e)=1$, it follows that $\operatorname{gcd}(C, D) \mid 4$. If $\operatorname{gcd}(C, D)=1$ or 4 , then $C$ and $D$ are squares. Thus $3 e^{2}-p M^{2}=n^{2},-p M^{2} \equiv n^{2}(\bmod 3), p \equiv-1(\bmod 3)$ and $p \equiv-1(\bmod 12)$.

If $\operatorname{gcd}(C, D)=2$, then $C / 2$ and $D / 2$ are squares. Thus $e^{2}+p M^{2}=2 n^{2}$, hence $e^{2} \equiv 2 n^{2}(\bmod p),\left(\frac{2}{p}\right)=1, p \equiv \pm 1(\bmod 8)$.

We proved that if $\# \alpha\left(\Gamma_{p}\right)=8$, then $p>3$ and either $p \equiv 1(\bmod 8)$, or $p \equiv-1$ $(\bmod 12)$. This proves (ii).

(iii) We have to estimate $\# \alpha\left(\bar{\Gamma}_{p}\right)$. It is clear that if $b_{1}<0$ then (6) has no solutions, and thus, by $b_{1} \mid 16 p^{2}$, we have $b_{1} \in\left\{2^{\alpha} p^{\beta}: 0 \leq \alpha \leq 4,0 \leq \beta \leq 2\right\}$. If $p=2$, then we obtain $\alpha\left(\bar{\Gamma}_{p}\right) \subset\{1,2\}\left(\bmod \mathbb{Q}^{* 2}\right)$. Therefore, we may assume $p>2$.

Let $b_{1}=2 p^{\beta}$, and suppose that (6) is solvable. Then $M$ is odd by $\operatorname{gcd}\left(M, 16 p^{2} / b_{1}\right)$ $=1$, and thus the left hand side of (6) is divisible by 4 , while the right hand side is not, which is impossible.

Next let $b_{1}=8 p^{\beta}$, and suppose that (6) is solvable. Then $N$ is even and, consequently, $e$ is odd. Thus the left hand side of (6) is divisible by 4 , while the right hand side is not, which is impossible. We obtain that $b_{1} \in\left\{1, p, p^{2}, 4,4 p, 4 p^{2}, 16,16 p, 16 p^{2}\right\}$ and $b_{1} \in\{1, p\}\left(\bmod \mathbb{Q}^{* 2}\right)$. This proves (iii).

(iv) Suppose that $\# \alpha\left(\bar{\Gamma}_{p}\right)=2$. Then $p \in \alpha\left(\bar{\Gamma}_{p}\right)$, and (6) is solvable for at least one of $b_{1}=p, b_{1}=4 p$ and $b_{1}=16 p$.

Let $b_{1}=p$, and suppose that $N^{2}=p M^{4}-4 p M^{2} e^{2}+16 p e^{4}$ is solvable. Then $M$ is odd by $\operatorname{gcd}(M, 16 p)=1$, and $N^{2} \equiv p M^{4}(\bmod 4)$. Hence $p>2$ and $p \equiv 1$ $(\bmod 4)$. We have $N=p N_{1}$ and

$$
p N_{1}^{2}=M^{4}-4 M^{2} e^{2}+16 e^{4}=\left(M^{2}-2 e^{2}\right)^{2}+12 e^{4}
$$

Now $p \nmid e$ by $\operatorname{gcd}\left(e, b_{1}\right)=1$, and we get $\left(\frac{-12}{p}\right)=1$. Since $p \equiv 1(\bmod 4)$, we obtain $\left(\frac{3}{p}\right)=1, p \equiv \pm 1(\bmod 12)$ and $p \equiv 1(\bmod 12)$.

The case $b_{1}=16 p$ is similar with the roles of $M$ and $e$ exchanged. Therefore, if (6) is solvable for $b_{1}=16 p$, then $p \equiv 1(\bmod 12)$.

Finally, let $b_{1}=4 p$, and suppose that $N^{2}=4 p M^{4}-4 p M^{2} e^{2}+4 p e^{4}$ is solvable. Then $2 \nmid M$ by $\operatorname{gcd}(M, 4 p)=1$, and $2 p \mid N$. Let $N=2 p N_{1}$, then $p N_{1}^{2}=M^{4}-$ $M^{2} e^{2}+e^{4}$. Since $M$ is odd, we have $M^{4}-M^{2} e^{2}+e^{4} \equiv 1(\bmod 4)$, and thus $p \equiv 1$ $(\bmod 4)$. We have

$$
4 p N_{1}^{2}=4 M^{4}-4 M^{2} e^{2}+4 e^{4}=\left(2 M^{2}-e^{2}\right)^{2}+3 e^{4} .
$$

Now $p \nmid e$ by $\operatorname{gcd}\left(e, b_{1}\right)=1$, and we get $\left(\frac{-3}{p}\right)=1$. Since $p \equiv 1(\bmod 4)$, we obtain $\left(\frac{3}{p}\right)=1, p \equiv \pm 1(\bmod 12)$ and $p \equiv 1(\bmod 12)$.

We proved that if $\# \alpha\left(\Gamma_{p}\right)=2$, then $p \equiv 1(\bmod 12)$. This proves (iv).

Our next aim is to prove

\section{Theorem 3.4}

(i) The rank of $\Gamma_{-p}$ is at most two for every positive prime $p$.

(ii) If $p \not \equiv 1(\bmod 12)$, then the rank of $\Gamma_{-p}$ is at most one.

(iii) If $p=2, p=3$ or $p \equiv 7(\bmod 24)$, then the rank of $\Gamma_{-p}$ is zero. 
We consider the curves

$$
C_{-p}: y^{2}=x^{3}-2 p x^{2}-3 p^{2} x \text { and } \bar{C}_{-p}: y^{2}=x^{3}+4 p x^{2}+16 p^{2} x .
$$

First we prove the following lemma.

\section{Lemma 3.5}

(i) $\# \alpha\left(\Gamma_{-p}\right) \leq 8$ for every prime $p$.

(ii) If $p=2, p=3$ or $p \equiv 7(\bmod 12)$, then $\# \alpha\left(\Gamma_{-p}\right) \leq 4$.

(iii) $\# \alpha\left(\bar{\Gamma}_{-p}\right) \leq 2$ for every prime $p$.

(iv) If $p \neq 3$ and $p \neq \equiv 1,13$ or $19(\bmod 24)$, then $\# \alpha\left(\bar{\Gamma}_{-p}\right)=1$.

Proof The proof of the statement (i) is the same as in the case of Lemma 3.3.

(ii) Suppose $\# \alpha\left(\Gamma_{-p}\right)=8$. As in the proof of (ii) of Lemma 3.3, this implies $p \neq 3$ and $-1 \in \alpha\left(\Gamma_{-p}\right)$. Therefore, by $b_{1} \mid-3 p^{2}, N^{2}=b_{1} M^{4}-2 p M^{2} e^{2}+\left(-3 p^{2} / b_{1}\right) e^{4}$ is solvable for at least one of $b_{1}=-1$ and $b_{1}=-p^{2}$.

Suppose that $N^{2}=-M^{4}-2 p M^{2} e^{2}+3 p^{2} e^{4}$ is solvable. If $p=2$, then $M$ is odd by $\operatorname{gcd}\left(M, 3 p^{2}\right)=1$, and $N^{2} \equiv-M^{4}(\bmod 4)$, which is impossible. If $p>3$, then $p \nmid M$ by $\operatorname{gcd}\left(M, 3 p^{2}\right)=1$, and thus we have $\left(\frac{-1}{p}\right)=1$ and $p \equiv 1(\bmod 4)$.

Next suppose that $N^{2}=-p^{2} M^{4}-2 p M^{2} e^{2}+3 e^{4}$ is solvable; then $\operatorname{gcd}(M, 3)=1$. If $p=2$, then $e$ is odd (since otherwise both $N$ and $e$ would be even), and $N^{2} \equiv 3 e^{4}$ $(\bmod 4)$, which is impossible. Suppose $p>3$. Then $p \nmid e$ by $\operatorname{gcd}\left(e, b_{1}\right)=1$, and thus $\left(\frac{3}{p}\right)=1$ and $p \equiv \pm 1(\bmod 12)$.

We have $N^{2}=\left(3 e^{2}+p M^{2}\right)\left(e^{2}-p M^{2}\right)=C \cdot D$. Since $p \nmid e$ and $\operatorname{gcd}(M, e)=1$, it follows that $\operatorname{gcd}(C, D) \mid 4$. If $\operatorname{gcd}(C, D)=1$ or 4 , then $C$ and $D$ are squares. Thus $3 e^{2}+p M^{2}=n^{2}, p M^{2} \equiv n^{2}(\bmod 3), p \equiv 1(\bmod 3)$ and $p \equiv 1(\bmod 12)$.

If $\operatorname{gcd}(C, D)=2$, then $C / 2$ and $D / 2$ are squares. Thus $3 e^{2}+p M^{2}=2 n^{2}$, hence $p \equiv p M^{2} \equiv 2 n^{2} \equiv 2(\bmod 3)$. Since $p \equiv \pm 1(\bmod 12)$, we get $p \equiv-1(\bmod 12)$.

We proved that if $\# \alpha\left(\Gamma_{-p}\right)=8$, then $p \equiv 1(\bmod 4)$ or $p \equiv-1(\bmod 12)$. This proves (ii).

(iii) The argument proving (iii) of Lemma 3.3 shows that $\alpha\left(\bar{\Gamma}_{-p}\right) \subset\{1, p\}\left(\bmod \mathbb{Q}^{* 2}\right)$.

(iv) Suppose $\# \alpha\left(\bar{\Gamma}_{-p}\right)=2$. Then $p \in \alpha\left(\bar{\Gamma}_{-p}\right)$, and

$$
N^{2}=b_{1} M^{4}+4 p M^{2} e^{2}+\left(16 p^{2} / b_{1}\right) e^{4}
$$

is solvable for at least one of $b_{1}=p, b_{1}=4 p$ and $b_{1}=16 p$.

Let $b_{1}=p$, and suppose that $N^{2}=p M^{4}+4 p M^{2} e^{2}+16 p e^{4}$ is solvable. Then $M$ is odd by $\operatorname{gcd}(M, 16 p)=1$, and $N^{2} \equiv p M^{4}(\bmod 4)$. Hence $p>2$ and $p \equiv 1$ $(\bmod 4)$. We have $N=p N_{1}$ and

$$
p N_{1}^{2}=M^{4}+4 M^{2} e^{2}+16 e^{4}=\left(M^{2}+2 e^{2}\right)^{2}+12 e^{4}
$$

Now $p \nmid e$ by $\operatorname{gcd}\left(e, b_{1}\right)=1$, and we get $\left(\frac{-12}{p}\right)=1$. Since $p \equiv 1(\bmod 4)$, we obtain $\left(\frac{3}{p}\right)=1, p \equiv \pm 1(\bmod 12)$ and $p \equiv 1(\bmod 12)$.

The case $b_{1}=16 p$ is similar with the roles of $M$ and $e$ exchanged. Therefore, if (6) is solvable for $b_{1}=16 p$, then $p \equiv 1(\bmod 12)$.

Finally, let $b_{1}=4 p$, and suppose that $N^{2}=4 p M^{4}+4 p M^{2} e^{2}+4 p e^{4}$ is solvable. Then $M$ is odd by $\operatorname{gcd}(M, 4 p)=1$. Also, $2 p \mid N$, and thus $e$ is odd. Let $N=2 p N_{1}$, 
then $p N_{1}^{2}=M^{4}+M^{2} e^{2}+e^{4}$. Thus $p N_{1}^{2} \equiv 3(\bmod 8)$, hence $p \equiv 3(\bmod 8)$. We have

$$
4 p N_{1}^{2}=4 M^{4}+4 M^{2} e^{2}+4 e^{4}=\left(2 M^{2}+e^{2}\right)^{2}+3 e^{4}
$$

Now $p \nmid e$ by $\operatorname{gcd}\left(e, b_{1}\right)=1$, and we get $p=3$ or $\left(\frac{-3}{p}\right)=1$. Suppose $p \neq 3$. Since $p \equiv 3(\bmod 4)$, we obtain $\left(\frac{3}{p}\right)=-1, p \equiv 5$ or $7(\bmod 12)$. Since $p \equiv 3(\bmod 8)$, we get $p \equiv 19(\bmod 24)$.

We proved that if $\# \alpha\left(\bar{\Gamma}_{-p}\right)=2$, then $p=3$ or $p \equiv 1(\bmod 12)$ or $p \equiv 19(\bmod$ 24), This proves (iv).

Proof of Theorem 3.4 Statements (i) and (ii) of the theorem follow from Lemma 3.5 and from (4). If $p=2$ or $p \equiv 7(\bmod 24)$, then the rank of $\Gamma_{-p}$ is zero by Lemma 3.5 and (4).

What remains to prove is that the rank of $\Gamma_{-3}$ is zero. Since $\# \alpha\left(\bar{\Gamma}_{-3}\right) \leq 2$ by Lemma 3.5, it is enough to show that $\# \alpha\left(\Gamma_{-3}\right) \leq 2$.

Consider the curve $C_{-3}: y^{2}=x^{3}-6 x^{2}-27 x$. Then $b_{1} \in\{ \pm 1, \pm 3, \pm 9, \pm 27\}$, and thus $\alpha\left(\Gamma_{-3}\right) \subset\{ \pm 1, \pm 3\}\left(\bmod \mathbb{Q}^{* 2}\right)$. We show that $3 \notin \alpha\left(\Gamma_{-3}\right)$. Suppose $3 \in \alpha\left(\Gamma_{-3}\right)$. Then the equation $N^{2}=b_{1} M^{4}-6 M^{2} e^{2}-\left(27 / b_{1}\right) e^{4}$ is solvable for at least one of $b_{1}=3$ and $b_{1}=27$.

Suppose that $N^{2}=3 M^{4}-6 M^{2} e^{2}-9 e^{4}$ is solvable. Then $3 \nmid M$ by $\operatorname{gcd}(M, 9)=1$, and $3 \nmid e$ since $3 \mid N$. Let $N=3 N_{1}$. Then $3 N_{1}^{2}=M^{4}-2 M^{2} e^{2}-3 e^{4}$, hence $M^{4} \equiv 2 M^{2} e^{2}(\bmod 3)$, which is impossible.

Finally, suppose that $N^{2}=27 M^{4}-6 M^{2} e^{2}-e^{4}$ is solvable. Then $3 \nmid e$ by $\operatorname{gcd}\left(e, b_{1}\right)=1$. Thus $N^{2} \equiv-e^{2}(\bmod 3)$, which is impossible.

Corollary 3.6 If $p=2, p=3$ or $p \equiv 7(\bmod 24)$, then the curves $C_{p}$ and $C_{-p}$ have no nontrivial rational points.

\section{Numerical Examples}

As the following table shows, for all primes $3<p<100$, if $p \not \equiv 7(\bmod 24)$, then at least one of the curves $C_{p}$ and $C_{-p}$ has nontrivial rational points and, consequently, $\Gamma_{p}$ or $\Gamma_{-p}$ has positive rank. Note that the point $(75,210)$ belongs to both $C_{-23}$ and $C_{73}$.

The points below were found by searching for integer solutions of $N^{2}=b_{1} M^{4} \pm$ $2 p M^{2} e^{2}+b_{2} e^{4}$ with $b_{1} b_{2}=-3 p^{2}$, and putting $x=b_{1} M^{2} / e^{2}, y=b_{1} M N / e^{3}$. The solutions for $p \neq 83$ were found by using GNU Octave (https://www.gnu.org/ software/octave/). I am grateful to Peter Salvi for finding a solution for $p=83$; he used Julia 1.0 (https://julialang.org/blog/2018/08/one-point-zero).

$$
\begin{aligned}
& p=5:(-1,8) \in \Gamma_{-5}, \\
& p=11:(75,720) \in \Gamma_{11}, \\
& p=13:(-12,90) \in \Gamma_{13}, \\
& p=17:(-1,30) \in \Gamma_{17},
\end{aligned}
$$




$$
\begin{aligned}
& p=19:\left(\frac{17689}{225}, \frac{1374688}{3375}\right) \in \Gamma_{-19}, \\
& p=23:(75,210) \in \Gamma_{-23} \text {, } \\
& p=29:\left(-\frac{529}{25}, \frac{16744}{125}\right) \in \Gamma_{-29} \text {, } \\
& p=37:\left(\frac{231361}{324}, \frac{116481365}{5832}\right) \in \Gamma_{37}, \\
& p=41:(-121,198) \in \Gamma_{41}, \\
& p=43:\left(\frac{4165798849}{21538881}, \frac{171543655606240}{99961946721}\right) \in \Gamma_{-43} \text {, } \\
& p=47:(1875,79050) \in \Gamma_{-47} \text {, } \\
& p=53:\left(-\frac{167281}{4225}, \frac{89165272}{274625}\right) \in \Gamma_{-53} \text {, } \\
& p=59:\left(-\frac{930433009}{6076225}, \frac{13189530387264}{14977894625}\right) \in \Gamma_{59}, \\
& p=61:(-108,1170) \in \Gamma_{61} \text {, } \\
& p=67:\left(\frac{909373939321}{51279921}, \frac{863887766632341760}{367215514281}\right) \in \Gamma_{-67}, \\
& p=71:(507,9282) \in \Gamma_{-71} \text {, } \\
& p=73:(75,210) \in \Gamma_{73} \text {, } \\
& p=83:\left(-\frac{2140232721200}{59682001401}, \frac{13897116923228469980}{14580253260262899}\right) \in \Gamma_{83} \text {, } \\
& p=89:\left(-\frac{121}{289}, \frac{489280}{4913}\right) \in \Gamma_{-89}, \\
& p=97:\left(-\frac{121}{25}, \frac{45408}{125}\right) \in \Gamma_{-97} \text {. }
\end{aligned}
$$

Acknowledgements Open access funding provided by Eötvös Loránd University (ELTE). The author was supported by the Hungarian National Foundation for Scientific Research, Grant No. K124749.

Open Access This article is distributed under the terms of the Creative Commons Attribution 4.0 International License (http://creativecommons.org/licenses/by/4.0/), which permits unrestricted use, distribution, and reproduction in any medium, provided you give appropriate credit to the original author(s) and the source, provide a link to the Creative Commons license, and indicate if changes were made.

\section{References}

1. Beeson, M.: Tiling an equilateral triangle. arXiv:1812.07014 (2018)

2. Beeson, M.: No triangle can be cut into seven congruent triangles. arXiv:1811.09723 (2018)

3. Laczkovich, M.: Tilings of triangles. Discrete Math. 140(1-3), 79-94 (1995)

4. Laczkovich, M.: Tilings of convex polygons with congruent triangles. Discrete Comput. Geom. 48(2), 330-372 (2012)

5. Silverman, J.H., Tate, J.: Rational Points on Elliptic Curves. Undergraduate Texts in Mathematics. Springer, New York (1992)

Publisher's Note Springer Nature remains neutral with regard to jurisdictional claims in published maps and institutional affiliations. 